\title{
COMPTON ECHOES FROM GAMMA-RAY BURSTS
}

\author{
Piero Madau, ${ }^{1,2}$ Roger D. Blandford, ${ }^{1,2,3}$ AND MARTIN J. ReES ${ }^{1}$ \\ Received 1999 December 14; accepted 2000 April 25
}

\begin{abstract}
Recent observations of $\gamma$-ray bursts (GRBs) have provided growing evidence for collimated outflows and emission and strengthened the connection between GRBs and supernovae. If massive stars are the progenitors of GRBs, the hard photon pulse will propagate in the preburst, dense environment. Circumstellar material will Compton scatter the prompt GRB radiation and give rise to a reflection echo. We calculate luminosities, spectra, and light curves of such Compton echoes in a variety of emission geometries and ambient gas distributions and show that the delayed hard X-ray flash from a pulse propagating into a red supergiant wind could be detectable by Swift out to $z \sim 0.2$. Independently of the $\gamma$-ray spectrum of the prompt burst, reflection echoes will typically show a high-energy cutoff between $m_{e} c^{2} / 2$ and $m_{e} c^{2}$ because of Compton downscattering. At fixed burst energy per steradian, the luminosity of the reflected echo is proportional to the beaming solid angle, $\Omega_{b}$, of the prompt pulse, while the number of bright echoes detectable in the sky above a fixed limiting flux increases as $\Omega_{b}^{1 / 2}$, i.e., it is smaller in the case of more collimated jets. The lack of an X-ray echo at about 1 month delay from the explosion poses severe constraints on the possible existence of a lateral GRB jet in SN 1987A. The late $r$-band afterglow observed in GRB 990123 is fainter than the optical echo expected in a dense red supergiant environment from an isotropic prompt optical flash. Significant $\mathrm{MeV}$ delayed emission may be produced through the bulk Compton (or Compton drag) effect resulting from the interaction of the decelerating fireball with the scattered X-ray radiation.
\end{abstract}

Subject headings: gamma rays: bursts — gamma rays: theory — supernovae: general $-\mathrm{X}$-rays: general

\section{INTRODUCTION}

The nature of the progenitors of $\gamma$-ray bursts (GRBs) remains an unsettled issue after more than three decades of research (e.g., Mészáros, Rees, \& Wijers 1999; Paczyński 1999; Piran 1999). The discovery of X-ray (Costa et al. 1997) and optical (van Paradijs et al. 1997) afterglows has provided the breakthrough needed to establish the cosmological nature of these events. While the implied huge energy release per steradian must produce a relativistically expanding fireball (e.g., Mészáros \& Rees 1992), it is not yet clear if this expansion is quasi-spherical or highly collimated, or if the degree of beaming differs between the prompt GRB and the delayed emission. The observed distribution of optical afterglows with respect to their host galaxies may suggest that some GRBs are associated with star-forming regions and therefore with the explosions of massive stars rather than with merging neutron stars (e.g., Paczyński 1998; Fruchter et al. 1999). Recent observations of a spatial and temporal coincidence between the supernova SN 1998bw and GRB 980425 (Galama et al. 1998) have added support to the idea that at least some GRBs may be related to some type of supernova explosion. The observation of a red component, a factor of 60 higher in flux than the extrapolated afterglow in GRB 980326, has also been explained with a supernova, and, if true, it would strengthen the connection of GRBs with massive stars (Bloom et al. 1999; see also Reichart 1999 for similar evidence in GRB 970228; but see Esin \& Blandford 2000 for an alternative explanation involving dust scattering). If GRBs are associated with

\footnotetext{
${ }^{1}$ Institute of Astronomy, Madingley Road, Cambridge CB3 OHA, England, UK.

${ }^{2}$ Institute for Theoretical Physics, University of California, Santa Barbara, CA 93106-4030.

${ }^{3}$ Theoretical Astrophysics, 130-33 Caltech, Pasadena, CA 91125.
}

$\mathrm{SN}$-like events, energetic considerations suggest that some fireballs be collimated into a solid angle $\Omega_{b} \ll 4 \pi$, i.e., GRBs involve strongly asymmetric outflows. Without beaming, the inferred ("isotropic-equivalent") energy of GRB 990123, $\sim 4 \times 10^{54}$ ergs (Kulkarni et al. 1999), rules out stellar models. Additional circumstantial evidence for jetlike bursts comes from the decline (" beaming break") observed in some afterglow light curves (Kulkarni et al. 1999; Harrison et al. 1999) and attributed to the sideways expansion of nonspherical ejecta, although some difficulties remain (Moderski, Sikora, \& Bulik 2000).

It is an unavoidable consequence of a massive star progenitor model for GRBs that the hard emission will propagate in a dense circumstellar environment, such as a preburst stellar wind (Chevalier \& Li 1999). Shells of significantly enhanced gas density may also exist in the immediate neighborhood of a GRB. This would be the case, for example, in scenarios in which a supernova occurs shortly before the burst (as in the delayed collapse of a rotationally stabilized neutron star; Vietri \& Stella 1998) so that the metal-enriched supernova remnant shell does not have time to disperse. The deceleration of a presupernova wind by the pressure of the surrounding medium could also create circumstellar shells, as would the interaction of fast and slow winds from massive stars (as observed in the case of SN 1987A). The ambient material will then efficiently scatter the prompt GRB radiation and, because of light-travel time effects, produce a luminous (albeit unresolvable) reflection echo. The detection of scattered light may provide unique information on the environment of GRBs and on their emission properties. Conversely, one may use the lack of evidence for a Compton echo to set constraints on the density of circumstellar material and the burst energetics. In this paper we compute the expected properties of such Compton flashes in a variety of scenarios and show that the delayed hard X-ray emission associated with the echo 
reflected by a red supergiant wind is significant and could be detectable by Swift out to a redshift of $z \sim 0.2$.

\section{COMPTON ECHOES FROM CIRCUMSTELLAR GAS}

For simplicity, we will approximate the prompt GRB as a collimated photon pulse that maintains a constant luminosity for a time $\Delta$. It is commonly proposed that the burst is produced when the kinetic energy of a relativistically expanding fireball is dissipated at a radius $r_{\mathrm{sh}} \sim 10^{13.5} \mathrm{~cm}$ because of, e.g., internal shocks, and radiated away as $\gamma$-rays through synchrotron emission. The scattered light is observed at a time $t \gg \Delta(t$ is measured from the time the burst is first detected), when the radiation beam can be visualized as a shell of radius $c t \gg r_{\mathrm{sh}}$ and thickness $c \Delta$. The (equal arrival time) scattering material lies on the paraboloid having the burst at its focus and its axis along the line of sight,

$$
r=\frac{c t}{1-\cos \theta},
$$

where $\theta$ is the angle between the line of sight and the direction of the reflecting gas as seen by the burst (e.g., Blandford \& Rees 1972).

The sudden brilliance of a GRB will be reflected by the circumstellar gas to create a "Compton echo." If $E$ is the total energy, $E \equiv \int E_{\epsilon \Omega} d \epsilon d \Omega$, emitted by the burst ( $E_{\epsilon \Omega}$ is the energy emitted per unit energy $\epsilon$ and unit solid angle $\Omega$ along the $\theta$ direction, $E_{\epsilon \Omega}=E_{\epsilon} / 4 \pi$, in the case of isotropic emission), then the equivalent isotropic luminosity (as inferred by a distant observer) of the echo reflected at a distance $r$ from the site of the burst is

$$
L_{\epsilon^{\prime}}=4 \pi \int n_{e}(r, \theta) E_{\epsilon \Omega} \frac{d \sigma}{d \Omega} \frac{d r}{d t} d \Omega,
$$

where the integral is over the beaming solid angle $\Omega_{b}$ of the prompt pulse, $r$ is given by equation (1),

$$
\frac{d \sigma}{d \Omega}=\frac{3 \sigma_{\mathrm{T}}}{16 \pi}\left(\frac{\epsilon^{\prime}}{\epsilon}\right)^{2}\left(\frac{\epsilon}{\epsilon^{\prime}}+\frac{\epsilon^{\prime}}{\epsilon}-\sin ^{2} \theta\right)
$$

is the differential Klein-Nishina cross section for unpolarized incident radiation (e.g., Rybicki \& Lightman 1979), $\sigma_{\mathrm{T}}$ is the Thomson cross section,

$$
\epsilon^{\prime}=\epsilon\left[1+\frac{\epsilon}{m_{e} c^{2}}(1-\cos \theta)\right]^{-1}
$$

is the energy of the scattered photon, and $n_{e}$ is the local electron density. Equation (2) assumes that photons scatter only once and that absorption can be neglected (which is a good approximation at observed energies $\gtrsim 10 \mathrm{keV}$ ).

In the relativistic regime, photons experience a reduced cross section and scatter preferentially in the forward direction. Electron recoil further suppresses the reflected echo at $\gamma$-ray energies. In the nonrelativistic regime, $\epsilon \approx \epsilon^{\prime}$, equation (3) reduces to the classical Thomson limit, and the observed echo luminosity becomes

$$
L_{\epsilon}=\frac{3}{4} \int n_{e} E_{\epsilon \Omega} \sigma_{\mathrm{T}} c \frac{1+\cos ^{2} \theta}{1-\cos \theta} d \Omega .
$$

Below we discuss a few scenarios in the Thomson regime that well illustrate the range of possible emission geometries and ambient gas distributions.

\subsection{Uniform Medium Extending to $r=R$}

1. Isotropic burst.-In this case the echo is dominated by gas along the line of sight, and

$$
L_{\epsilon}=\frac{3}{4} n_{e} E_{\epsilon} \sigma_{\mathrm{T}} c\left[\ln \left(\frac{2 R}{c t}\right)-\left(1-\frac{c t}{2 R}\right)^{2}\right]
$$

for $t<2 R / c$. The emission diverges logarithmically at zero lag and then decreases monotonically to zero at $t=2 R / c$.

2. Collimated burst.- - Here the zone of emission propagates out along the approaching and receding jets (assumed to have equal energy $E_{\epsilon} / 2$ ) until $r=R$, and

$$
\begin{aligned}
& L_{\epsilon}=\frac{3}{4} n_{e} E_{\epsilon} \sigma_{\mathrm{T}} c \\
& \quad \times \begin{cases}\frac{1+\cos ^{2} \theta}{1-\cos ^{2} \theta} & \text { if } 0<t<R(1-\cos \theta) / c, \\
\frac{1+\cos ^{2} \theta}{2(1+\cos \theta)} & \text { if } R(1-\cos \theta) / c<t<R(1+\cos \theta) / c,\end{cases}
\end{aligned}
$$

where $\theta$ is the angle between the line of sight and the approaching beam.

\subsection{Constant-Velocity Wind with $N_{e}=A r^{-2}$}

1. Isotropic burst.-The reflected flash comes from the surface of the paraboloid and is dominated by the apex behind the source,

$$
L_{\epsilon}=\frac{A E_{\epsilon} \sigma_{\mathrm{T}}}{c t^{2}} .
$$

The echo declines with time faster than most observed afterglows.

2. Collimated burst.-In this case

$$
L_{\epsilon}=\frac{3 A E_{\epsilon} \sigma_{\mathrm{T}}}{4 c t^{2}}\left(1+\cos ^{2} \theta\right),
$$

and the echo is dominated by the receding jet as, at a given observer time, it originates closer to the GRB where the density is greater.

\subsection{Thin Spherical Shell of Radius $R$ and Column $N_{e}$}

This scenario may arise if the progenitor star loses most of its mass quite rapidly a short but finite time prior to the explosion.

1. Isotropic burst.- One derives

$$
L_{\epsilon}=\frac{3 N_{e} E_{\epsilon} \sigma_{\mathrm{T}} c}{4 R}\left(1-\frac{c t}{R}+\frac{c^{2} t^{2}}{2 R^{2}}\right)
$$

for $c t<2 R$. As the paraboloid sweeps up the shell, the echo luminosity decreases, reaches a minimum when $t=R / c$, and increases again until the back of the shell is passed by the paraboloid.

2. Collimated burst.- In this case the echo light curve is the sum of two delta functions,

$$
\begin{aligned}
L_{\epsilon}= & \frac{3}{8} N_{e} E_{\epsilon} \sigma_{\mathrm{T}}\left(1+\cos ^{2} \theta\right) \\
& \left\{\delta\left[\frac{t-R(1-\cos \theta)}{c}\right]+\delta\left[\frac{t-R(1+\cos \theta)}{c}\right]\right\} .
\end{aligned}
$$




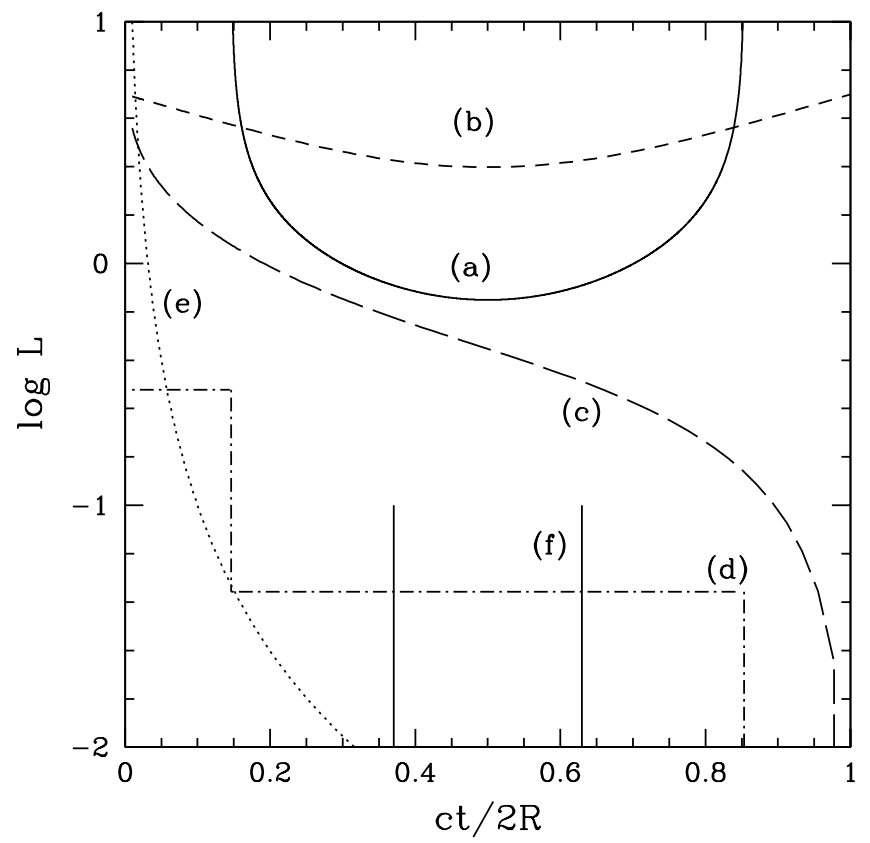

FIG. 1.-Possible X-ray flux variations observed following a GRB and due to Thomson scattering in the circumstellar medium. A variety of ambient gas distributions have been assumed (see text for details): the scattered fluxes, expressed as equivalent isotropic luminosities, have arbitrary normalizations. Solid curve $(a)$ : slender annular ring of radius $R$, inclined at $45^{\circ}$ to the line of sight; isotropic burst. Short-dashed line $(b)$ : thin spherical shell of radius $R$; isotropic burst. Long-dashed line $(c)$ : uniform density sphere of radius $R$; isotropic burst. Dash-dotted line $(d)$ : uniform density sphere of radius $R$, collimated burst inclined at $45^{\circ}$ to the line of sight. Dotted line $(e)$ : constant-velocity wind $\left(n_{e} \propto r^{-2}\right)$. Double spike $(f)$ : thin spherical shell of radius $R$; burst is collimated into two antiparallel beams inclined at $75^{\circ}$ to the line of sight.

The two spikes of emission are seen separated by an interval $2 R \cos \theta / c$.

\subsection{Slender Annular Ring of Radius $R$ and Angular Width $\Delta \theta$}

This model is inspired by observations of SN 1987A and $\eta$ Carinae, both of which exhibit dense equatorial rings. Let the ring have an inclination $i$. The echo associated with an isotropic burst then has luminosity

$$
L_{\epsilon}=\frac{3 N_{e} E_{\epsilon} \Delta \theta \sigma_{\mathrm{T}} c}{4 \pi R} \frac{\left(1-c t / R+c^{2} t^{2} / 2 R^{2}\right)}{\left(2 c t / R-c^{2} t^{2} / R^{2}-\cos ^{2} i\right)^{1 / 2}},
$$

where

$$
\frac{R(1-\sin i)}{c}<t<\frac{R(1+\sin i)}{c} .
$$

The emission is maximized at the beginning and at the end of the response. The echo temporal behavior in some of the scenarios discussed above is shown in Figure 1.

\section{WIND MODELS}

In the following we will assume a broken power law for the "typical" GRB spectrum,

$$
\epsilon E_{\epsilon} \propto \begin{cases}\epsilon & \text { if } \epsilon \leq 250 \mathrm{keV}, \\ \epsilon^{-0.25} & \text { if } \epsilon>250 \mathrm{keV} .\end{cases}
$$

This is consistent with a recent analysis of $\sim 150$ spectra obtained by the Burst and Transient Source Experiment (BATSE) on the Compton Gamma Ray Observatory (Preece et al. 2000). No attempt has been made to correct the observed break energy for the mean redshift of the GRB population.

\subsection{Radiative Acceleration of Ambient Material}

Equation (2) only applies to a scattering medium that is either at rest or moving with subrelativistic speed. As shown by Madau \& Thompson (2000) and Thompson \& Madau (2000), a strong burst of radiation will have important dynamical effects on the surrounding interstellar medium. Optically thin material overtaken by an expanding photon shell at radius $r$ will develop a large bulk Lorentz factor $\Gamma$ when the energy deposited by Compton scattering exceeds the rest-mass energy of the scatterers, i.e., when

$$
\mathscr{S} \equiv \frac{E \bar{\sigma}}{\Omega_{b} r^{2} \mu_{e} m_{p} c^{2}} \gg 1,
$$

where $\Omega_{b}$ is the burst beaming angle, $\mu_{e}$ is the molecular weight per electron, $m_{p}$ is the proton mass, and $\bar{\sigma}$ is the spectrum-weighted total cross section,

$$
\bar{\sigma}=E^{-1} \int E_{\epsilon} \frac{d \sigma}{d \Omega} d \Omega d \epsilon \approx 0.2 \sigma_{\mathrm{T}},
$$

from equations (3) and (13). In the case of a pulse of "isotropic-equivalent" energy $E\left(4 \pi / \Omega_{b}\right)=10^{53}$ ergs, propagating into a medium with $\mu_{e}=2$, one can then define a characteristic distance

$$
r_{c}(\mathscr{S} \sim 1) \approx 6 \times 10^{14} \mathrm{~cm}\left(\frac{4 \pi E}{10^{53} \Omega_{b} \text { ergs }}\right)^{1 / 2},
$$

such that for $r \ll r_{c}$ dynamical effects become important and may suppress the scattering rate (by a factor of $\sim 1-\beta)$.

More quantitatively, consider a plane-parallel photon pulse of thickness $c \Delta$ propagating into the circumstellar gas. A parcel of matter moving radially with speed $c \beta=d r / d t$ will be accelerated at the rate

$$
\frac{d \Gamma}{d r}=\Gamma^{2}(1-\beta)^{2} \frac{\tilde{\mathscr{S}}}{c \Delta},
$$

where now $\tilde{\mathscr{S}}$ includes a correction to $\bar{\sigma}$ as particles moving at relativistic speed scatter an increasing fraction of (redshifted) photons with the full Thomson cross section (i.e., $\bar{\sigma} \rightarrow \sigma_{\mathrm{T}}$ ). Matter initially at radius $r$ and accelerated from rest will surf the photon shell over a distance $\Delta r$ such that

$$
c \Delta=\int_{r}^{r+\Delta r} d r \frac{1-\beta}{\beta} .
$$

The radiative force vanishes when the photon shell moves past the particle at $r+\Delta r$. When $\Delta r \ll r$, the inverse square dilution of flux can be neglected, and equation (18) can be rewritten using equation (17) as

$$
\tilde{\mathscr{S}}=\int_{1}^{\Gamma_{\max }} \frac{d \Gamma}{\Gamma^{2} \beta(1-\beta)} .
$$

This can be integrated exactly to yield a maximum Lorentz factor

$$
\Gamma_{\max }=\cosh [\ln (1+\tilde{\mathscr{S}})],
$$

which decreases with distance from the source. In the relativistic limit $\Gamma_{\max } \rightarrow \tilde{\mathscr{S}} / 2$ (Madau \& Thompson 2000). The 
acceleration distance is

$$
\Delta r=\frac{c \Delta}{\tilde{\mathscr{S}}} \int_{1}^{\Gamma_{\max }} \frac{d \Gamma}{\Gamma^{2}(1-\beta)^{2}}=c \Delta \frac{\tilde{\mathscr{S}}}{2}\left(1+\frac{\tilde{\mathscr{S}}}{3}\right) .
$$

Figure 2 shows the bulk Lorentz factor derived from a numerical integration (including Klein-Nishina corrections) of equation (17) for a burst of isotropic-equivalent energy $10^{53}$ ergs, duration $\Delta=10 \mathrm{~s}$, and spectrum as in equation (13). As expected, the acceleration at large radii takes place on a distance $\Delta r \ll r / c$, and the outflow becomes subrelativistic at about $r_{c}$. Two effects must be noted here. First, the accelerated medium will be compressed into a shell of thickness $\sim r / \Gamma_{\text {max }}^{2}$. Shocks may form when inner shells (which move faster and are more compressed) run into outer shells, and material will accumulate at $r_{c}$. In an $r^{-2}$ density profile, the electron scattering optical depth in the wind from $r_{c}$ to infinity is $\tau_{c}=n_{e}\left(r_{c}\right) \bar{\sigma} r_{c}$. The mass accumulated at $r_{c}$ by a burst of energy $E_{\Omega}$ per steradian is

$$
\frac{E_{\Omega} \tau_{c}}{c^{2}}=0.006 M_{\odot} \operatorname{sr}^{-1}\left(\frac{E_{\Omega}}{10^{52} \mathrm{ergs} \mathrm{sr}^{-1}}\right) \tau_{c} .
$$

Preacceleration of the ambient medium by the prompt radiation pulse will slow down the deceleration of the fireball ejecta (Thompson \& Madau 2000). Second, we have solved the equations above assuming the ambient medium to be

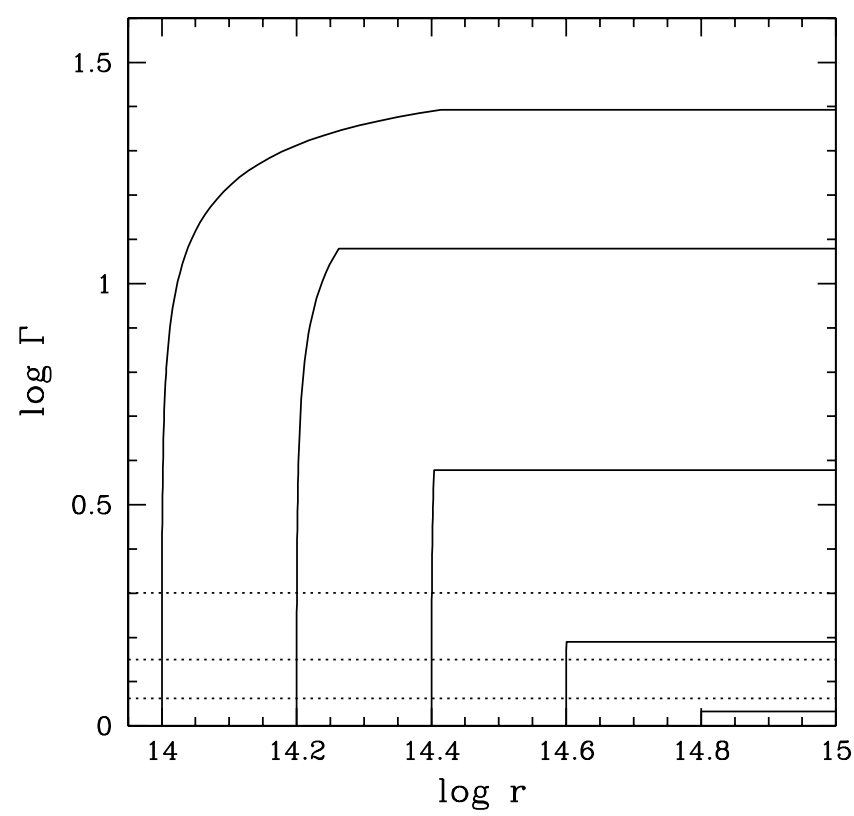

FIG. 2.-Bulk Lorentz factor of optically thin, circumstellar material with molecular weight per electron $\mu_{e}=2$, as a function of Lagrangian distance $r$ (in $\mathrm{cm}$ ) from a collimated source of impulsive radiation. The photon pulse (assumed to be plane-parallel) has an "isotropic-equivalent" energy of $10^{53} \mathrm{ergs}$, duration $10 \mathrm{~s}$, and spectrum as in eq. (13). The radiative force vanishes when the photon shell moves past the particle. The equation of motion has been integrated in the Klein-Nishina regime assuming the material to be initially at rest at $\log r=14,14.2,14.4,14.6$, and $14.8 \mathrm{~cm}$ (solid curves). The accelerated medium will be compressed into a shell of thickness $\sim r / \Gamma^{2}$. Shocks may form when inner shells (which move faster and are more compressed) run into outer shells, and material will accumulate at a radius $r_{c} \sim 6 \times 10^{14} \mathrm{~cm}$ where the outflow becomes subrelativistic. The three dotted lines show the value of $\Gamma$ for which $\beta=\cos \theta$, where $\theta=\left(30^{\circ}, 45^{\circ}, 60^{\circ}\right)$ is the angle between the line of sight and the approaching jet of a collimated burst. Above these lines the scattered radiation will be "beamed away" from the line of sight. composed of a baryonic plasma. It has been shown by Thompson \& Madau (2000) that $e^{+} e^{-}$pair creation occurs in GRBs when side-scattered photons collide with the main $\gamma$-ray beam, an effect which amplifies the density of scattering charges in the ambient medium. The pair density will exponentiate when the photon shell is optically thick to photon collisions, i.e., when $\tau_{\gamma \gamma} \approx n_{\gamma} \sigma_{\mathrm{T}} c \Delta / 4 \sim 1$. As $\tau_{\gamma \gamma} \approx$ $\mathscr{S} \mu_{e} m_{p} c^{2} /\langle\epsilon\rangle$, runaway pair production may occur well beyond the radius $r_{c}$ defined in equation (16). When pairs are produced in sufficient numbers, i.e., when $2 m_{e} n_{e^{+}} \gg$ $m_{p} n_{p}$, the mean mass per scattering charge drops to $\sim m_{e}$. Because of the reduced inertia per particle, and also because pair-producing collisions impart direct momentum to the gas, such a pair-loaded plasma may, under some circumstances, be more efficiently accelerated to relativistic bulk velocities than a baryonic gas. This could increase the value of $r_{c}$ in equation (16) by as much as a factor of $\left(\mu_{e} m_{p} / m_{e}\right)^{1 / 2}$. On the other hand, runaway pair creation will also boost the scattering optical depth of circumstellar material, thus producing brighter echoes at later times. For simplicity, in the rest of this paper we will limit our discussion to a baryonic scattering medium at $r \gtrsim r_{c}$, where dynamical effects can be neglected. We defer a proper treatment of reflected echoes in an $e^{+} e^{-}$pair-dominated wind to another work.

\subsection{Red Supergiant Winds}

In the case of a massive progenitor scenario, such as a "collapsar" (MacFadyen \& Woosley 1999) or "hypernova" (Paczyński 1998), it is known that red supergiants and Wolf-Rayet (W-R) stars have strong winds. If the progenitor is a Type Ib or Type Ic SN, then it must have lost its hydrogen and perhaps its helium envelope at some earlier time. The winds from typical red supergiants are slow moving and dense, with velocities $v_{w} \approx 10-20 \mathrm{~km} \mathrm{~s}^{-1}$ and mass-loss rates between $10^{-6}$ and $10^{-4} M_{\odot} \mathrm{yr}^{-1}$.

As a "representative" red supergiant wind, consider the case of SN 1993J. While in a steady, spherically symmetric wind, the electron density drops as

$$
n_{e}(r)=\frac{\dot{M}}{4 \pi v_{w} r^{2} \mu_{e} m_{p}} .
$$

Deviations from an $r^{-2}$ density gradient toward a flatter slope, $n_{e} \propto r^{-1.5}$, have been inferred in the circumstellar medium of this supernova by Fransson, Lundquist, \& Chevalier (1996) and are possibly caused by a variation of the mass-loss rate from the progenitor or by a nonspherical geometry. Following Fransson et al. (1996), one can write

$$
\begin{aligned}
n_{e}(r) \approx & 10^{8} \mathrm{~cm}^{-3}\left(\frac{r}{10^{15} \mathrm{~cm}}\right)^{-1.5} \\
& \times\left(\frac{\dot{M}}{4 \times 10^{-5} M_{\odot} \mathrm{yr}^{-1}}\right)\left(\frac{v_{w}}{10 \mathrm{~km} \mathrm{~s}^{-1}}\right)^{-1}
\end{aligned}
$$

up to $r=2 \times 10^{16} \mathrm{~cm}$, while at larger radii the observations appear to be consistent with an $r^{-2}$ law.

Figure 3 shows the reflected echo at $t=8,24$, and $72 \mathrm{hr}$ of a two-sided GRB jet inclined at an angle $\theta$ to the line of sight, propagating through an SN 1993J-like dense environment. The prompt pulse was assumed to radiate a total of $10^{52}$ ergs with the spectrum given in equation (13), each jet having equal strength and being invisible to the observer. It is instructive to look at the relative contribution of the approaching and receding beams. On the equal 

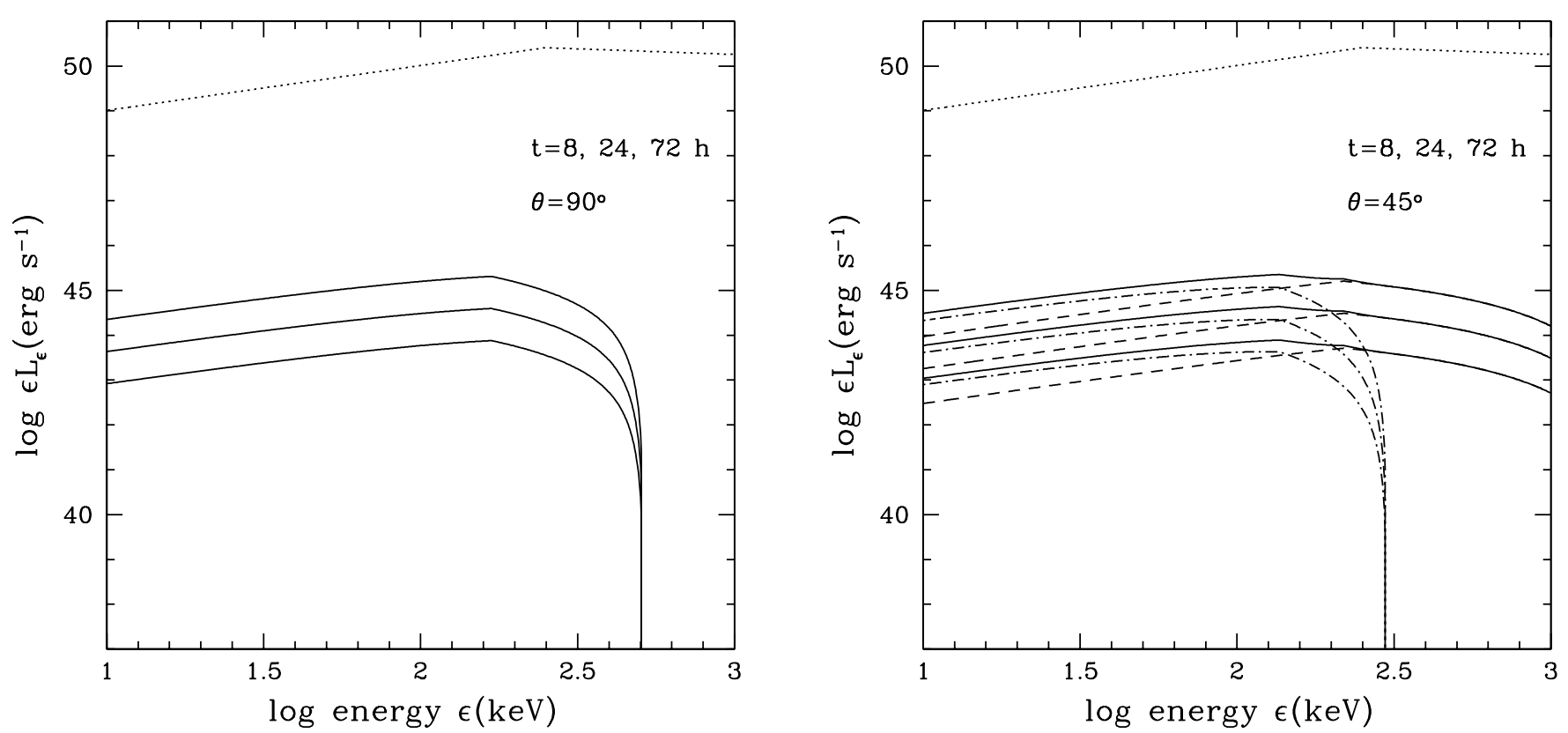

FIG. 3. - Compton echo of a GRB. The primary burst is assumed to be a two-sided collimated pulse of total energy $10^{52}$ ergs and duration $10 \mathrm{~s}$ (dotted spectrum, top of each panel), propagating at an angle $\theta$ with the line of sight and invisible to the observer. The circumstellar red supergiant wind has an $r^{-1.5}$ density profile up to $2 \times 10^{16} \mathrm{~cm}$, steepening to a -2 slope at larger radii. The electron density is normalized to $n_{e}=10^{8} \mathrm{~cm}{ }^{-3}$ at $r=10^{15} \mathrm{~cm}$. Top: $\theta=90^{\circ}$. Solid lines: observed echo at $t=8,24$, and $72 \mathrm{hr}$ after the burst (from top to bottom). Bottom: $\theta=45^{\circ}$. Solid lines: same as before. Dashed lines: reflected light from approaching beam. Dash-dotted lines: reflected light from receding beam.

arrival time paraboloid, the receding beam is reflected by gas that is closer to the source and denser: its contribution dominates the echo at all energies where scattering occurs in the Thomson regime. Above $150 \mathrm{keV}$, however, recoil can no longer be neglected, and it is the approaching beam (whose photons are seen after small-angle scattering) that dominates the reflected flash at high energies. The total spectral energy distribution therefore mirrors the prompt burst at low energies but is much steeper beyond a few

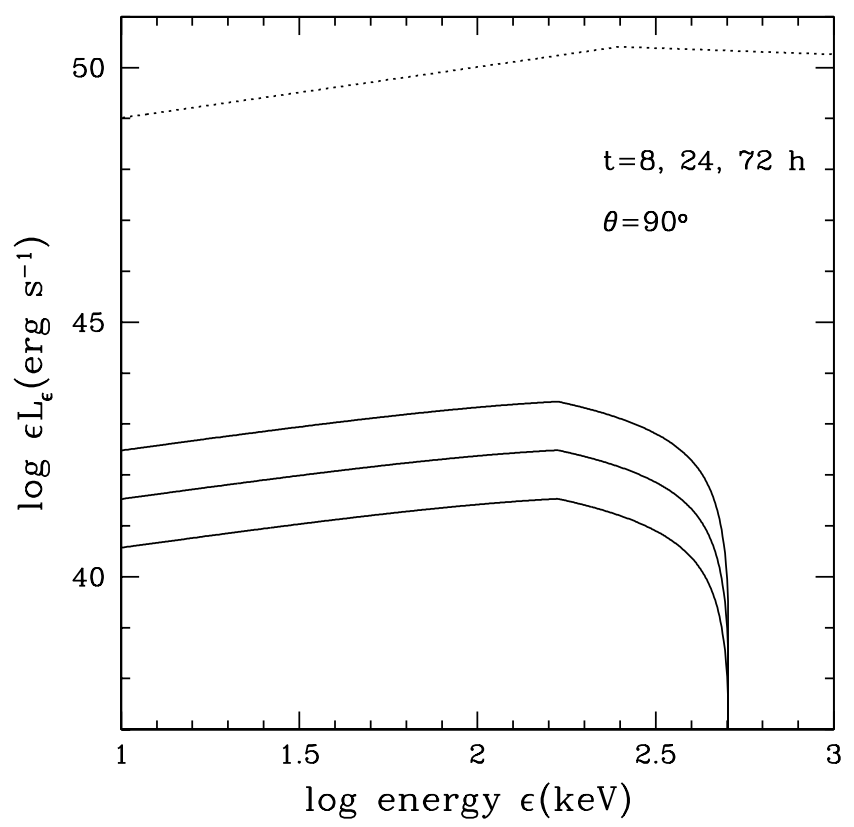

hundred $\mathrm{keV}$. In the limit $\theta=90^{\circ}$ both beams are detected in reflected light after wide-angle scattering, and the echo is suppressed above $511 \mathrm{keV}$ by Compton downscattering as $\epsilon^{\prime} \rightarrow m_{e} c^{2} /(1-\cos \theta)$ for $\epsilon \gg m_{e} c^{2}$. The scattered luminosity in Figure 3 drops initially as $t^{-1.5}$ for $c t<2 \times 10^{16}(1-\cos \theta) \mathrm{cm}$ and then steepens to $t^{-2}$ first at $\gamma$-ray energies (when the approaching beam encounters the $r^{-2}$ density profile) and only later (depending on the jet angle) at X-ray energies (dominated by the receding beam).

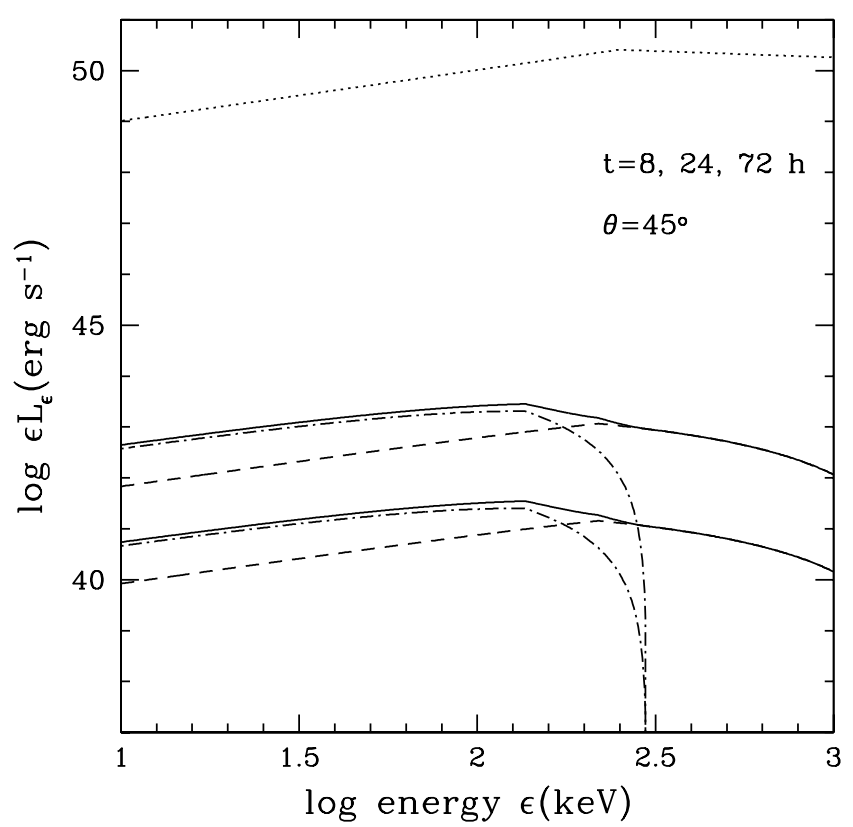

FIG. 4.- Same as Fig. 3, except that the circumstellar W-R wind has an $r^{-2}$ density profile with electron density $n_{e}=1.5 \times 10^{6} \mathrm{~cm}^{-3}$ at $r=10^{15} \mathrm{~cm}$. The scattered luminosity drops as $t^{-2}$ in all cases. 


\subsection{Wolf-Rayet Winds}

The winds from W-R stars are characterized by mass-loss rates $\dot{M} \approx 10^{-5}$ to $10^{-4} M_{\odot} \mathrm{yr}^{-1}$ and velocities $v_{w} \approx$ $1000-2500 \mathrm{~km} \mathrm{~s}^{-1}$ (e.g., Willis 1991). In a steady, spherically symmetric wind, the electron density is

$$
\begin{aligned}
n_{e}(r) \approx & 3 \times 10^{6} \mathrm{~cm}^{-3}\left(\frac{r}{10^{15} \mathrm{~cm}}\right)^{-2} \\
& \times\left(\frac{\dot{M}}{10^{-4} M_{\odot} \mathrm{yr}^{-1}}\right)\left(\frac{v_{w}}{1000 \mathrm{~km} \mathrm{~s}^{-1}}\right)^{-1} \mu_{e}^{-1},
\end{aligned}
$$

where $\mu_{e} \sim 2$ in a helium gas. Figure 4 shows the fainter (compared to the red supergiant wind case) Compton echo of a GRB jet (with the same parameters as above) propagating through a W-R wind.

In the case of an $r^{-2}$ medium with the fiducial scalings given in equations (16) and (25), the electron scattering optical depth from $r_{c}$ to infinity is $\tau_{c} \approx 3 \times 10^{-4}$. This is roughly the fraction of GRB energy that is reflected in the echo on all timescales $t \gtrsim r_{c} / c \sim 6 \mathrm{hr}$, with a scattered luminosity that drops as $t^{-2}$. Fast-moving winds will be less dense and therefore less efficient $\left(10^{-5} \lesssim \tau_{c} \lesssim 10^{-3}\right)$ at reprocessing the prompt pulse; slow-moving winds will produce brighter echoes $\left(10^{-4} \lesssim \tau_{c} \lesssim 0.05\right)$. As the electron-scattering optical depth is $\tau_{c} \propto r_{c} \propto\left(E / \Omega_{b}\right)^{-1 / 2}$, fainter and less collimated bursts will be characterized by a larger "albedo" relative to bright ones. For an isotropic burst of energy, say $E=10^{50}$ ergs, the characteristic distance in equation (16) decreases to $r_{c} \approx 2 \times 10^{13} \mathrm{~cm}$. In a red supergiant wind with $n_{e}\left(r_{c}\right) \approx 4 \times 10^{10} \mathrm{~cm}^{-3}$, one has $\tau_{c} \approx 0.2$, and a fraction $\left(1-e^{-\tau_{c}}\right) \sim 20 \%$ of the GRB energy will be reflected in an echo on timescales $\gtrsim 10$ minutes.

\section{DISCUSSION}

Bright scattering echoes are a natural consequence of a hard photon pulse propagating in a dense circumstellar environment such as a preburst stellar wind. In massive-star progenitor models for GRBs there is likely to be an echo component in the observed X-ray and $\gamma$-ray light curves; the only question is how significant this component is. In the range $10-100 \mathrm{keV}$, where they mirror the spectral energy distribution of the prompt pulse, echoes will typically be harder than the afterglows observed by BeppoSAX. Above $200 \mathrm{keV}$, the reflected flash will have a much steeper spectrum than the parent GRB as scattering occurs in the relativistic regime. While, in the standard fireball/blast wave scenario, both the prompt and delayed emission may be highly beamed, Compton echoes are (modulo the scattering phase function) quasi-isotropic. Backscattered radiation could then provide a means for detecting a population of nearby misaligned GRBs since collimated outflows imply the existence of a large amount of undetected dim bursts and a much higher event rate than is often assumed. In a $\gamma$-ray-quiet burst, the observed luminosity of a Compton echo at fixed burst energy per steradian is proportional to the beaming solid angle, $L \propto \Omega_{b}$, as it scales with the intrinsic power of the parent GRB. In the Euclidean (bright) part of the number-flux relation the total number of echoes above a fixed limiting flux then scales as $L^{3 / 2} \Omega_{b}^{-1} \propto \Omega_{b}^{1 / 2}$, i.e., it is smaller in the case of more collimated jets. In the flat (faint) part of the counts, instead, the number of echoes in the sky is approximately independent of the beaming solid angle.

Compton echoes could be studied with the Swift GammaRay Burst Explorer, to be launched in about 2003. Swift will detect and follow GRBs with the Burst and Alert Telescope (BAT) at energies in the 10-100 keV range, together with X-ray (XRT) and optical (UVOT) instrumentation (Gehrels 1999). Long-duration $\gamma$-ray emission from the burst will be studied simultaneously with the X-ray and optical afterglow emission. With a sensitivity of 2 mcrab in a $16 \mathrm{hr}$ exposure, the BAT on board Swift will detect a $10^{45.7} \mathrm{ergs} \mathrm{s}^{-1}$ echo out to a distance of $1 \mathrm{Gpc}(z \sim 0.2)$.

\subsection{Echoes versus $X$-Ray Afterglows}

It is interesting to compare the expected energetics of Compton echoes with the observed X-ray late afterglows. In a cosmology with $H_{0}=65 \mathrm{~km} \mathrm{~s}^{-1} \mathrm{Mpc}^{-1}, \Omega_{M}=0.3$, and $\Omega_{\Lambda}=0.7$, the (isotropic-equivalent) luminosity of GRB 9702283 days after the event was $\sim 4 \times 10^{44} \mathrm{ergs} \mathrm{s}^{-1}$ in the $2-10 \mathrm{keV}$ band, with a decay rate $\propto t^{-1.3}$ (Costa et al. 1997; Djorgovski et al. 1999).

Let us assume, for simplicity, that both the prompt and delayed GRB emission are isotropic. Had GRB 970228 ( $E \approx 10^{52}$ ergs) occurred in an SN 1993J-like environment (eq. [24]), the expected light echo from an isotropic pulse,

$$
\epsilon L_{\epsilon} \approx 4 \times 10^{44} \mathrm{ergs} \mathrm{s}^{-1}\left(\frac{E}{10^{52} \mathrm{ergs}}\right) t_{\text {days }}^{-1.5}\left(\frac{\epsilon}{10 \mathrm{keV}}\right),
$$

would be slightly fainter at $10 \mathrm{keV}$ than the observed 2-10 $\mathrm{keV}$ afterglow. At a $t=10 \mathrm{hr}$ delay its $100 \mathrm{keV}$ luminosity would exceed $10^{46} \mathrm{ergs} \mathrm{s}^{-1}$, still consistent with the OSSE upper limit of Matz et al. (1997). A break in the echo light curve to a $t^{-2}$ decline would be expected at $t \approx 2 \times 10^{16}$ $\mathrm{cm} / c \sim 8$ day delay. Based on equation (26), we conclude that the scattered radiation from a pulse propagating in a red supergiant wind has a flux that is comparable to the observed $X$-ray late afterglows. The echo would be about 2 orders of magnitude fainter in a W-R-type wind on account of the greater speed. Note that the light emitted during the early afterglow will also be scattered by circumstellar material and will give rise to a light echo; as the luminosity decays with time, however, "later" paraboloids will be sequentially dimmer, that is, for any given radial distance from the burst the reflected power will actually decrease.

\section{2. $X$-Ray Delayed Outbursts}

A delayed outburst was observed in the X-ray afterglow of GRB 970508. The event had a luminosity of $\sim 2 \times 10^{45}$ $\mathrm{ergs} \mathrm{s}^{-1}$ (Piro et al. 1998; Metzger et al. 1997) at $9 \mathrm{hr}$ delay, with a decay $\propto t^{-1.1}$ up to $6 \times 10^{4} \mathrm{~s}$. This was followed by a second flare of activity with a duration of approximately a few times $10^{5} \mathrm{~s}$. The excess energy was a significant fraction of the total, and the spectrum became harder during the flare (a possible detection of redshifted iron-line emission has also been reported by Piro et al. 1999). The outburst could be explained by the Compton echo from a thin circumstellar shell of enhanced gas density in the neighborhood of the GRB. For an isotropic burst the reflected luminosity from the shell remains constant to within a factor of 2 (see Fig. 1, curve $b$ ). While emission from the afterglow shock would then dominate over the scattered 
radiation at early times because of its steeper light curve, this may not be necessarily true at later times. For a shell radius of $R=1.5 \times 10^{15} \mathrm{~cm}$ (assumed to be larger than the distance reached by the shock producing the underlying afterglow emission), thickness $R / 5$, and Thomson optical depth $\tau_{\mathrm{T}}=0.2$, one derives a mean density ${ }^{4} n_{e} \approx 5 \times 10^{8}$ $\mathrm{cm}^{-3}$ and a total mass of $0.007 M_{\odot}\left(\mu_{e}=2\right)$. The echo reaches a maximum at $t_{\text {max }}=2 R / c \approx 10^{5} \mathrm{~s}$, with luminosity

$$
\begin{aligned}
\epsilon L_{\epsilon}= & \frac{3 \tau_{\mathrm{T}} \epsilon E_{\epsilon}}{2 t_{\max }} \\
& \approx 7 \times 10^{44} \mathrm{ergs} \mathrm{s}^{-1}\left(\frac{E}{10^{52} \text { ergs }}\right) t_{\text {max }, \text { days }}^{-1} \\
& \times\left(\frac{\epsilon}{10 \mathrm{keV}}\right)
\end{aligned}
$$

(see eq. [10] and Fig. 1), enough to outshine the power-lawdecaying afterglow at late times. The spectrum in the flare would be harder as it mirrors the prompt emission. A temporary brightening should be observed again at later times as the afterglow shock reaches the shell and passes in front of it.

\section{3. $\mathrm{MeV}$ Delayed Emission}

We have shown in $\S 3.2$ (see also Figs. 2 and 3 ) that Compton downscattering will produce a high-energy cutoff in the echo $\gamma$-ray emission at energies between $m_{e} c^{2} / 2$ and $m_{e} c^{2}$. There is a competing effect, however, that may generate $\mathrm{MeV}$ photons at late times. While, in the standard fireball/blast wave scenario, the source of X-ray and $\gamma$-ray radiation is itself expanding at relativistic speed and photons are beamed into a narrow angle along the direction of motion, the light scattered off circumstellar material is quasi-isotropic and can interact with the relativistic ejecta via the bulk (inverse) Compton effect (or Compton drag). In an $r^{-2}$ surrounding medium that is either at rest or moving at subrelativistic speed, the energy density $U$ of the reflected radiation drops as $r^{-4}$, and the effect will be dominated by the inner regions close to the characteristic distance $r_{c}$. The scattered energy density at X-ray frequencies is

$$
U_{\mathrm{X}}=\frac{L_{\mathrm{X}}}{4 \pi r_{c}^{2} c}=\frac{E_{\mathrm{X}} n_{e} \sigma_{\mathrm{T}}}{4 \pi r_{c}^{2}}
$$

(isotropic burst). As an illustrative possibility, consider a relativistic fireball made up by an individual shell of instantaneous bulk Lorentz factor $\Gamma_{F}$, and let the scattering charges be cold in the fluid frame, $\left\langle\gamma_{e}\right\rangle \sim 1$. Seed photons of energy $\epsilon_{\mathrm{X}}$ will then be upscattered to energies $\sim \epsilon_{\mathrm{X}} \Gamma_{F}^{2}$ if $\epsilon_{\mathrm{X}} \Gamma_{F} \ll m_{e} c^{2}$, or to $m_{e} c^{2} \Gamma_{F}$ otherwise. At $t=r_{c} / c \sim$ a few hours, the shock interaction of the relativistic ejecta with the circumstellar wind may have already slowed down the fireball to $\Gamma_{F} \sim$ a few. If $E$ is the total (initial) energy of the fireball, the instantaneous emitted power from bulk

\footnotetext{
${ }^{4}$ This is a factor of 10 higher than the characteristic density of a red supergiant wind at this distance (see eq. [24]), an enhancement that could be caused by a variation of the mass-loss rate from the progenitor, by interacting slow and fast winds, by condensations formed via cooling instabilities, or by runaway $e^{+} e^{-}$pair creation induced by collisions between soft side-scattered radiation and the main $\gamma$-ray photon beam.
}

Compton scattering can be written as

$$
\mathscr{L}_{e}\left(\epsilon_{\mathrm{X}} \Gamma_{F}^{2}\right) \approx\left(\frac{E}{m_{p} c^{2} \Gamma_{F}}\right)\left(\frac{\Gamma_{F}^{2} E_{\mathrm{X}} n_{e} \sigma_{\mathrm{T}}^{2} c}{4 \pi r_{c}^{2}}\right),
$$

where the first term in parentheses is the number of particles being Compton dragged at that instant (assuming the shock evolves adiabatically), and the second term is the rate of inverse Compton losses in the Thomson limit. The luminosity received at Earth is $\mathscr{L}_{r} \approx 2 \Gamma_{F}^{2} \mathscr{L}_{e}$ as a result of the Doppler contraction of the observed time. With $E=10^{53}$ ergs, $\epsilon_{\mathrm{X}}=40 \mathrm{keV}, E_{\mathrm{X}}=4 \times 10^{51}$ ergs, $r_{c}=10^{15} \mathrm{~cm}, \Gamma_{F}=$ 5 , and $n_{e}=10^{7} \mathrm{~cm}^{-3}$, one derives $\mathscr{L}_{r}(1 \mathrm{MeV}) \sim 7 \times 10^{47}$ $\operatorname{ergs~s}^{-1}$ at an observed time $r_{c} /\left(2 \Gamma_{F}^{2} c\right) \sim 10$ minutes. These numbers are only meant to be indicative, since they depend on the uncertain evolution of the relativistic ejecta. Note that, in the case of a burst propagating in a circumstellar wind, the pressure of the scattered photons will not be able to compete with the external material in braking the fireball and therefore will not dictate its time evolution (see Lazzati et al. 2000 for a different scenario). For the opposite to be true the radiation energy density $U_{\mathrm{X}}$ would have to exceed the rest-mass energy density of the scatterers. This would drive a relativistic outflow, suppressing the scattering rate and leading to an inconsistency.

\subsection{A Lateral GRB Jet from $S N 1987$ A?}

It has recently been suggested by Cen (1999) that the bright, transient companion spot to SN 1987A observed about 1 month after the explosion (Nisenson et al. 1987; Meikle, Matcher, \& Morgan 1987) may have been caused by a receding GRB jet traveling at $\theta=127^{\circ}$ with respect to the SN-to-observer direction, through a circumstellar medium with a stellar wind-like density $n_{e} \propto r^{-2}$. The scenario proposed by Cen has $n_{e}=1 \mathrm{~cm}^{-3}$ at $r \approx 10^{19} \mathrm{~cm}$, an "isotropic-equivalent" burst energy $4 \pi E / \Omega_{b}=2 \times 10^{54}$ ergs, and a beaming angle $\Omega_{b}=1.5 \times 10^{-3} \mathrm{rad}$. The late optical emission produced in an external shock model by synchrotron radiation appears then to provide an adequate explanation for the evolution of the observed companion spot. If the jet had approached us along the line of sight, a very bright GRB would have been observed instead.

With these parameters and the GRB spectrum given in equation (13), one would expect from equation (5) a hard X-ray echo of luminosity

$$
\epsilon L_{\epsilon} \approx 10^{42} \operatorname{ergs~s}^{-1}\left(\frac{E}{10^{50} \text { ergs }}\right) t_{\text {days }}^{-2}\left(\frac{\epsilon}{20 \mathrm{keV}}\right) .
$$

From 2 to 8 weeks after the explosion, however, no significant flux in the 10-30 keV band was observed by Ginga in the direction of SN 1987A to a crude upper limit of $10^{37}$ ergs s$^{-1}$ (Dotani et al. 1987; Makino 1987). The lack of a detectable Compton echo therefore places severe constraints on the brightness of a possible GRB jet associated with SN 1987A, as only an unusually weak burst of intrinsic energy $E \lesssim$ a few times $10^{47}$ ergs would be compatible with the assumed circumstellar density profile.

\subsection{Optical Echoes}

A strong prompt optical flash accompanied the brightest burst seen by BeppoSAX, GRB 990123. The flash was observed by the Robotic Optical Transient Search Experiment (ROTSE) while the burst was still in progress, reached 
a peak of 9th magnitude, and then decayed with a powerlaw slope of -2 (Akerlof et al. 1999). The redshift of this burst ( $z=1.6$; Kelson et al. 1999) implies a peak luminosity of $5 \times 10^{49}$ ergs s$^{-1}$ and a total optical energy of $E_{\mathrm{opt}} \approx 2$ $\times 10^{51}$ ergs. An isotropic optical flash of this brightness, occurring in an SN 1993J-like dense stellar wind, would give rise to an optical light echo of luminosity

$$
\left(\epsilon L_{\epsilon}\right)_{\mathrm{opt}} \approx 10^{45} \mathrm{ergs} \mathrm{s}^{-1}\left(\frac{E_{\mathrm{opt}}}{2 \times 10^{51} \mathrm{ergs}}\right) t_{\mathrm{days}}^{-1.5} .
$$

Here we have assumed that the opacity is dominated by electron scattering, since the prompt flash will photoionize the ambient medium and destroy any dust by thermal sublimation out to a radius of $\sim 1$ pc (Waxman \& Draine 2000). However, beyond this radius the refractory cores of dust grains can survive until they are passed by the expanding blast wave. These grains have high albedo, selective extinc- tion, and forward scattering and may scatter the GRB light from the first few hours to form a supernova-like optical echo after a few months (Esin \& Blandford 2000).

Two days after the event, the transient afterglow was observed in the $r$ band at a level of $7 \mu \mathrm{Jy}$ (Kulkarni et al. $1999)$, or $\left(\epsilon L_{\epsilon}\right)_{r} \approx 8 \times 10^{43}$ ergs s$^{-1}$, quite a bit fainter than the expected optical echo. The data would then appear to rule out a dense red supergiant environment for GRB 990123 unless the prompt optical flash is actually beamed, which it may be.

We have benefited from many useful discussions with $\mathrm{G}$. Ghisellini, D. Helfand, E. Ramirez-Ruiz, and C. Thompson. Support for this work was provided by the NSF through grant PHY94-07194 (P. M. and R. D. B.), by NASA through grant 5-2837 and the Beverly and Raymond Sackler Foundation (R. D. B.), and by the Royal Society (M. J. R.).
Akerlof, C., et al. 1999, Nature, 398, 400

Blandford, R. D., \& Rees, M. J. 1972, Astrophys. Lett., 10, 77

Bloom, J. S., et al. 1999, Nature, 401, 453

Cen, R. 1999, ApJ, 524, L51

Chevalier, R. A., \& Li, Z.-Y. 1999, ApJ, 520, L29

Costa, E., et al. 1997, Nature, 387, 783

Djorgovski, S. G., et al. 1999, GCN Rep. 289

Dotani, T., et al. 1987, Nature, 330, 230

Esin, A. A., \& Blandford, R. D. 2000, ApJ, 534, L15

Fransson, C., Lundquist, P., \& Chevalier, R. A. 1996, ApJ, 461, 993

Fruchter, A. S., et al. 1999, ApJ, 520, 54

Galama, T. J., et al. 1998, Nature, 395, 670

Gehrels, N. 1999, BAAS, 31, 993

Harrison, F. A., et al. 1999, ApJ, 523, L121

Kelson, D. D., Illingworth, G. D., Franx, M., Magee, D., \& van Dokkum, P. G. 1999, IAU Circ. 7096, 3

Kulkarni, S. R., et al. 1999, Nature, 398, 389

Lazzati, D., Ghisellini, G., Celotti, A., \& Rees, M. J. 2000, ApJ, 529, L17

MacFadyen, A., \& Woosley, S. E. 1999, ApJ, 524, 262

Madau, P., \& Thompson, C. 2000, ApJ, 534, 239

Makino, F. 1987, IAU Circ. 4336, 1

Matz, S. M., et al. 1997, IAU Circ. 6578, 1

Meikle, W. P. S., Matcher, S. J., \& Morgan, B. L. 1987, Nature, 329, 608

Mészáros, P., \& Rees, M. J. 1992, ApJ, 397, 570

\section{REFERENCES}

Mészáros, P., Rees, M. J., \& Wijers, R. A. M. J. 1999, NewA, 4, 303

Metzger, M. R., et al. 1997, IAU Circ. 6676, 3

Moderski, R., Sikora, M., \& Bulik, T. 2000, ApJ, 529, 151

Nisenson, P., Papaliolios, C., Karovska, M., \& Noyes, R. 1987, ApJ, 320, L15

Paczyński, B. 1998, ApJ, 494, L45

1999, in The Largest Explosions since the Big Bang: Supernovae and Gamma-Ray Bursts, ed. M. Livio, K. Sahu, \& N. Panagia (Cambridge: Cambridge Univ. Press), in press

Piran, T. 1999, Phys. Rep., 314, 575

Piro, L., et al. 1998, A\&A, 331, L41 1999, ApJ, 514, L73

Preece, R. D., et al. 2000, ApJS, 126, 19

Reichart, D. E. 1999, ApJ, 521, L111

Rybicki, G. B., \& Lightman, A. P. 1979, Radiative Processes in Astrophysics (New York: Wiley)

Thompson, C., \& Madau, P. 2000, ApJ, 538, 105

van Paradijs, J., et al. 1997, Nature, 386, 686

Vietri, M., \& Stella, L. 1998, ApJ, 507, L45

Waxman, E., \& Draine, B. T. 2000, ApJ, 537, 796

Willis, A. J. 1991, in Wolf-Rayet Stars and Interrelations with Other Massive Stars in Galaxies, ed. K. A. van der Hucht \& B. Hidayat (Dordrecht: Kluwer), 256 